\title{
Use and misuse of emergency medical services by neurological patients presenting to a Greek Tertiary Health Care facility
} Emmanuil Dermitzakis*, Jobst Rudolf, Ioannis Gravas, Panagiotis Kyriakidis, Chrysanthi Bouziani, Georgios Georgiadis and Iakovos Tsiptsios

Address: Department of Neurology, General Hospital "Papageorgiou", Thessaloniki, Greece

* Corresponding author

from International Society on Brain and Behaviour: 3rd International Congress on Brain and Behaviour

Thessaloniki, Greece. 28 November - 2 December 2007

Published: 17 April 2008

Annals of General Psychiatry 2008, 7(SuppI I):SI76 doi:I0.I I86/I744-859X-7-SI-SI 76

This abstract is available from: http://www.annals-general-psychiatry.com/content/7/SI/SI76

(c) 2008 Dermitzakis et al.; licensee BioMed Central Ltd.

\section{Background}

With a population of about 1,000,000 inhabitants, Metropolitan Thessaloniki is the second largest town in Greece and the capital of the region of Macedonia. Among the city's 12 public hospitals, Papageorgiou General Hospital is one of its four tertiary health care facilities. In the metropolitan areas of Athens and Thessaloniki, public hospitals are on-call not on a daily, but on a one-in-four-days rotation basis. This system leads to an immense number of patients that presents every day to the Emergency Outpatient Departments (EOD) of Greek public hospitals, but exact data on the use (and maybe misuse) of public health facilities remain scarce.

\section{Materials and methods}

In a prospective observational study, demographic and medical data were collected from all patients presenting for a presumed neurological disorder to the EOD of Papageorgiou General Hospital, with special respect to the use of public emergency transport services (PETS). The study covered the time period from 1.2.2006 to 31.1.2007.

\section{Results}

The absolute number of patients examined for a presumed neurological disorder during the study period was 5901. Final diagnosis was stroke (all subtypes) in 36.3\%, vertigo in $13.4 \%$, episodic loss of consciousness in $9.6 \%$, psychiatric disorder in $9.4 \%$, epileptic fits in $7.9 \%$, headache in $4.2 \%$, stupor or coma in $4.2 \%$ and other diagnosis in $15.0 \%$ of the patients. From this total of 5901 patients,
$1242(21,0 \%)$ used PETS for their transportation to the EOD. In summer (June to August), PETS were used by $17.9 \%$ of the patients, while this percentage rose to $22.8 \%$ in winter (November-January). Only 705 (56.8\%) of these 1242 patients that used PETS for transportation were eventually admitted to a hospital ward.

\section{Conclusions}

The Greek National Health System is hospital-based. The paucity of public primary health care facilities forces patients to present immediately to a secondary or tertiary health care facility. Inevitably, this results in a misuse of PETS that becomes evident from the fact that more than $40 \%$ of the patients presenting to the EOD using PETS were not even admitted to hospital. Thus, misuse of PETS increases the financial burden of the Greek National Health System. 